\title{
Correction Note
}

G. Sankaranarayanan and C. Suyambulingom ${ }^{1}$ ): Distribution of the Maximum of the Number of Impulses at Any Instant in a Type II Counter in a Given

Interval of Time.

Metrika, vol. 18, 227-233, 1972

Page 227 delete 25 th line and insert "independent of the instants of arrivals and the events of realisation of the impulses".

In equation (8) and (10) in page 230 replace $h(u)$ by $f(u)$.

In equation (11) in page 230 in both terms replace $f(v)$ by $h(v)$.

In equation (18) in page 231 in all the three integrals delete $\mathrm{e}^{-\lambda v}$.

Delete equation (19) in page 231.

Number the 20 th line of the page 233 as equation (20), insert the upper limit ' $t$ ' in the second integral and in it replace 2 in the exponent by ' $v$ '.

In equation (21) of page 231 replace $n$ by ' $u$ '.

Delete equations (22), (23), (26) and (27).

Replace equation (24) in page 232 by

$$
s \theta_{k}^{\prime}(s)=-\lambda \theta_{k}^{\prime}(s+\mu)+\frac{\lambda^{2}}{\lambda+s+2 \mu} \theta_{k-1}^{\prime}(s+2 \mu)
$$

Replace equation (25) of page 232 by

$$
E \theta_{k}^{\prime}(s)=\theta_{k}^{\prime}(s+\mu)
$$

In equation (28) replace $E_{2}$ by $E$.

In the line 10 in page 233 insert

$$
t_{1}=\phi_{k-1}^{\prime}(s)=\lambda^{2} E^{2}\left(\frac{\theta_{k-1}^{\prime}(s)}{s+\lambda}\right) .
$$

1) Prof. Dr. G. Sankaranarayanan and C. Suyambulingom, Department of Mathematics and Statistics, Annamalai University, Annamalainagar, South India. 